\title{
Impacto do pastoreio bovino em florestas nebulares no Parque Nacional de São Joaquim, Santa Catarina
}

\author{
Effects of cattle gazing on cloud forests in São Joaquim National Park, Santa \\ Catarina state, Brazil
}

\author{
Ricardo de Vargas Kilca ${ }^{\mathrm{I}}$, Pedro Higuchi" ${ }^{\mathrm{II}}$, Ana Carolina da Silva ${ }^{\mathrm{II}}$
}

\begin{abstract}
Resumo
A pecuária é a atividade econômica muito comum do Planalto Catarinense e, talvez por isso, poderia ser a que desempenha a maior perturbação nos remanescentes de florestas nebulares (FN). Frente à abrangência deste impacto e a inexistência de estudos que avaliem a magnitude desta perturbação, foram investigadas as mudanças na composição florística e estrutura do componente arbóreo em uma cronossequência das FN em diferentes níveis de intensidade de pastoreio (fraco, médio, forte e com abandono) em áreas privadas não desapropriadas do Parque Nacional de São Joaquim, Urubici - SC. Oito parcelas de $1.000 \mathrm{~m}^{2}$ foram instaladas nas FN (seis áreas próximas e dentro de um mesmo fragmento de encosta e duas áreas de floresta em estágio inicial fora deste fragmento) e amostradas todas as espécies arbóreas (arbustos e árvores) em duas classes de tamanho (Cl.1 = Alt $>0,2 \mathrm{~m}$ e DAP $<1,9 \mathrm{~cm}$ e Cl.2 = DAP $\geq 1,9 \mathrm{~cm}$ ). A maioria das espécies arbóreas foi tolerante aos níveis baixos e intermediários de pastoreio e, por isso, poucos padrões florísticos foram revelados entre as florestas secundárias, exceto nos estandes bem jovens e os mais antigos. De forma geral, o pastoreio intenso no sub-bosque alterou mais a composição e estrutura do componente regenerativo (Cl.1) do que as plantas já estabelecidas (Cl.2). No entanto, o uso da restauração passiva parece ser suficiente para recuperação das FN não fortemente impactadas pelo pastoreio no Planalto Catarinense.
\end{abstract}

Palavras-chave: Floresta de altitude; Impacto ambiental; Estágio sucessional; Restauração passiva

\begin{abstract}
The cattle grazing is a common economic activity on montane forests (MFs) in subtropical highlands of Santa Catarina state, southern Brazil. There is no study on this impact on MFs. The aim of this study was to investigate changes in floristic and structure in the MFs( 1,700m.a.s.l) with different successional and levels of impact of cattle grazing in farms not expropriated located in São Joaquim National Park. Six plots with $1000 \mathrm{~m}^{2}$ were installed in the same mountain side (small variations in environmental conditions among sites) and two areas around the mountain. All arboreal component (shrubs and trees) in two classes $(\mathrm{A}=$ height $>0,2 \mathrm{~m}$ e $\mathrm{DBH}<1,9 \mathrm{~cm}$ e $\mathrm{B}=\mathrm{DBH} \geq 1,9 \mathrm{~cm})$ were sampled. Most arboreal species of MFs tend to be tolerant of low and intermediate levels of cattle grazing. Species richness was higher in MFs with low and medium intensity impact grazing. Most other structural attributes varied their response to grazing levels and successional stage of the forest. In general, the intense grazing in understory changed over the composition and structure of the regenerative component (Alt $>0.2 \mathrm{~m}$ and DAP $<1.9 \mathrm{~cm}$ ) than with larger plants. To promote sustainable grazing systems in Legal Reserve Areas, many studies are still needed. However, passive restoration has proven effective to restore cloud forests which suffer the impact of with cattle grazing.
\end{abstract}

Keywords: Montane tropical forests; Environmental impact; Successional stage; Passive restoration

Biólogo, Dr., Professor Adjunto da Universidade Estadual do Rio Grande do Sul, Unidade São Borja, Av. Presidente Tancredo Neves, 210, Bairro Piraí, CEP 97670-000, São Borja (RS), Brasil. ricardo-kilca@uergs.edu.br (ORCID: 0000-0003-1085-7641)

Engenheiros Florestais, Professores Adjuntos do Departamento de Engenharia Florestal, Centro Agroveterinário, Av. Luiz de Camões, 2090, CEP 88520-000, Lages (SC), Brasil. higuchip@gmail.com (ORCID: 0000-0002-3855-555X) / ana.carolina@udesc.br (ORCID: 0000-0002-1285-640X) 


\section{Introdução}

As florestas de altitude são também denominadas de florestas nebulares por estarem a maior parte do ano cobertas por nuvens, apresentarem alta umidade, menores luminosidade incidente e temperaturas do que as florestas nas altitudes menores (WEBSTER, 1995). Esses fatores físicos singulares determinam a estrutura, composição e fisionomiatípica destas formações florestais (FOSTER, 2001). Dentro do cenário global, as florestas nebulares são consideradas ameaçadas devido às elevadas taxas de deflorestamento e a suscetibilidade às mudanças climáticas (PONCE-REYES et al., 2013). Além das características físicas do ambiente, as mudanças no estágio sucessional e as perturbações alteram estrutura e composição destas formações florestais (MARTIN et al., 2011).

No sul do Brasil, as florestas nebulares são classificadas como fitofisionomias do bioma Mata Atlântica e denominadas de Floresta Ombrófila Densa Altomontana ou Floresta Ombrófila Mista Altomontana quando ocorrem a partir dos 1.000 m.n.m (IBGE, 2012). Apresentam-se em regiões de temperaturas baixas, solos rasos, úmidos e pobres em nutrientes e, por isso, destacamse das demais fitofisionomias florestais em menores altitudes por ter menor diversidade, árvores de menor altura, troncos retorcidos, maior densidade de fustes, dossel uniforme e denso sem árvores emergentes, folhas pequenas e, frequentemente, coriáceas e a abundância de epífitas avasculares (RODERJAN, 2002; FALKENBERG, 2003; SCHEER et al., 2011).

A região do Planalto Catarinense se destaca por apresentar grandes áreas de florestas de altitudes no sul do Brasil. No entanto, os estudos sobre estas formações florestais iniciaram a pouco mais de 10 anos (FALKENBERG, 2003) e, até então, poucos estudos foram realizados (FALKENBERG; VOLTOLINI, 1995; FALKENBERG, 2003; MARTINS et al., 2011; SCHEER et al., 2011; HIGUCHI et al., 2013; MARCON et al., 2014). A criação de bovinos é uma atividade econômica antiga na região e, por isso, o impacto do pastoreio destaca-se pela grande abrangência nas florestas nebulares, que por sua vez, servem de abrigo e fonte de alimentação e dessedentação para o gado ao longo do ano (FALKENBERG, 2003). O efeito do pastoreio bovino no subbosque das florestas nebulares é evidente, tanto na redução da regeneração natural, na seleção de espécies tolerantes e intolerantes ao pastejo, na estrutura e na dinâmica destas formações (FALKENBERG, 2003). Contudo, nenhum estudo quantitativo foi delineado para elucidar o impacto do pastoreio nas florestas nebulares que poderia indicar ou não sustentabilidade desta prática econômica em áreas de reserva legal (BRASIL, 2012) para então traçar estratégias para conservação, recuperação e uso racional destes ecossistemas (BUBB et al., 2004).

O Parque Nacional de São Joaquim foi criado em 1961 e dentro da sua área prevista de 49.800 hectares, apenas 36,4\% da área apresenta regularização fundiária (FAXINA, 2014). Assim, os proprietários que aguardam a regularização de suas glebas têm como fonte de renda principalmente a pecuária. Falkenberg (2003) destacou a existência frequente de pastoreio bovino nas florestas nebulares de toda a região dos Aparados da Serra em especial no PARNASJ. Portanto, este estudo tem como objetivo avaliar o impacto do pastoreio bovino nas mudanças florísticas e estruturais em florestas nebulares em diferentes estágios sucessionais e intensidades de impacto nas áreas não regularizadas do PARNA-SJ. Os resultados permitirão avaliar possíveis indicadores ambientais de impacto ambiental, inferir sobre a adaptabilidade das espécies arbóreas em diferentes níveis de pastoreio, avaliar a eficiência da restauração passiva na recuperação das florestas degradadas pelo pastoreio bovino e demonstrar o estado de conservação das florestas nebulares que poderão fazer parte do PARNA-SJ. 


\section{Materiais e métodos}

\section{Caracterização da área de estudo}

O Parque Nacional de São Joaquim (PARNA-SJ) está localizado na região sul do Estado de Santa Catarina, abrangendo os municípios de Urubici (Sede Administrativa), Bom Jardim da Serra, Orleans e Grão Pará (ver limites do PARNA-SJ em BRASIL, 2016). Apresenta uma área aproximada de 49.300 ha e um perímetro de $114 \mathrm{~km}$, com altitudes entre 350 e 1.822 metros. No PARNA-SJ podem ser encontradas as Unidades Geomorfológicas dos Patamares da Serra Geral (faixa estreita e descontínua associada à dissecação da rede de drenagem) e da Serra Geral (com relevo declivoso formando canyons com rochas expostas e pouca vegetação). As três principais classes de solos no PARNA-SJ são o Cambissolo, o Nitossolo e o Neossolo Litólico (SOUZA, 2004).

O clima predominante é o mesotérmico médio, caracterizado por temperatura média inferior a $10^{\circ} \mathrm{C}$ pelo menos em um mês do ano, não apresentando período de seca. A média do mês mais quente (janeiro) é de $20^{\circ} \mathrm{C}$, e $6^{\circ} \mathrm{C}$ no mês mais frio (julho). Os três fenômenos climáticos mais característicos são a neve e a geada no inverno e o nevoeiro ao longo do ano (SOUZA, 2004). De acordo com a classificação do IBGE (2012), pode-se encontrar dentro do PARNA-SJ as seguintes tipologias vegetais: Floresta Ombrófila Densa Submontana, Floresta Ombrófila Densa Montana, Floresta Ombrófila Densa Alto-Montana, Floresta Ombrófila Mista Montana, Floresta Ombrófila Mista Alto-Montana e os Campos. As florestas nebulares avaliadas se situam em propriedades privadas dentro do Parque Nacional de São Joaquim (PARNA-SJ) que ainda não foram desapropriadas.

\section{Desenho amostral}

Para a escolha dos estandes (ou áreas amostrais) em diferentes estágios sucessionais e níveis de impacto por pastoreio nas florestas nebulares no PARNA-SJ foram consideradas entrevistas com antigos funcionários do PARNA-SJ e antigos moradores (para conhecer o tipo de distúrbio e o histórico de uso/abandono), imagens orbitais recentes do Google Earth e referências bibliográficas. Posteriormente, foram realizadas saídas de campo (ano de 2015) para checar as informações com as constatações in loco. Para diminuir as variações ambientais no espaço, foram escolhidos, predominantemente, seis trechos de florestas nebulares em uma mesma encosta (vertente leste-norte), próximas do Morro da Igreja, em altitude similar (1.700 a 1.780 m.n.m), predominantemente numa mesma classe de solo (Cambissolo), fisionomia uniforme da vegetação e que apresentassem diferentes estágios sucessionais e níveis de perturbação por pastoreio bovino. Todos os trechos estão distantes entre si dezenas de metros (Tabela 1). Outros dois estandes de florestas nebulares foram incluídos na amostragem porque são bastante representativos na paisagem regional e não foram representados na área da encosta avaliada: uma área em estágio inicial de regeneração (iniciação) originada de antigos campos pastoreados que foram abandonados (12 anos sem pastoreio) e uma área de campo fortemente pastoreado, na qual está presente uma floresta nebular fortemente impactada. Desta forma, a amostragem procurou representar o maior número possível de condições ambientais em estágios sucessionais e nível de impacto por pastoreios ocorrentes nas florestas nebulares da região da Serra Catarinense. Os critérios de classificação dos estágios sucessionais foram adaptados da resolução CONAMA 4/94 (BRASIL, 1994) e de Frelich (2016) (Tabela 1). Foram estabelecidos os seguintes níveis de impacto por pastoreio bovino (Tabela 1): A) abandono/sem registro de pastoreio - sub-bosque denso (regeneração natural e pequenas árvores) e sem registros visuais de caminhamento e fezes bovinas(1A1) ou apenas trilhas antigas de caminhamento (4A8); B) pouco pastoreio sub-bosque com pouca vegetação (regeneração natural e pequenas árvores) e poucos registros

Ci. Fl., Santa Maria, v. 30, n. 1, p. 1-17, jan./mar. 2020 
visuais de trilhas por caminhamento animal e fezes; C) médio pastoreio - sub-bosque com pouca vegetação (regeneração natural e pequenas árvores), mas registros visuais frequentes de trilhas de caminhamento e de fezes em toda a área; D) pastoreio intenso - sub-bosque praticamente sem vegetação, apenas árvores adultas, registro de trilhas ativas por caminhamento e fezes frequentes em toda a área.

Tabela 1 - Trechos de florestas nebulares em diferentes estágios de sucessão e níveis de pastoreio bovino na região do Parque Nacional São Joaquim - SC

Table 1 - Cloud Forests in different levels of regeneration and cattle grazing in São Joaquin National Park, Santa Catarina state, Brazil

\begin{tabular}{|c|c|c|c|c|c|}
\hline Código & Alt. & Coordenadas & Solos & Ambiente & Declividade \\
\hline 1A1 & 1650 & $\begin{array}{c}28^{\circ} 06^{\prime} 46^{\prime \prime} \mathrm{S} \text { e } \\
49^{\circ} 29^{\prime} 52^{\prime \prime} \mathrm{O}\end{array}$ & Cambissolo & Campo abandonado & SO \\
\hline 2D5 & 1620 & $\begin{array}{c}28^{\circ} 06^{\prime} 38^{\prime \prime} \mathrm{S} \mathrm{e} \\
49^{\circ} 30^{\prime} 03^{\prime \prime} \mathrm{O}\end{array}$ & Cambissolo & Campo com floresta & SO \\
\hline 2D3 & 1740 & $\begin{array}{l}28^{\circ} 07^{\prime} 00^{\prime \prime} \mathrm{S} \mathrm{e} \\
49^{\circ} 29^{\prime} 57^{\prime \prime} \mathrm{O}\end{array}$ & Cambissolo & Floresta em encosta & $\mathrm{O}$ \\
\hline 2D4 & 1750 & $\begin{array}{c}28^{\circ} 07^{\prime} 01^{\prime \prime} \mathrm{S} \text { e } \\
49^{\circ} 30^{\prime} 00^{\prime \prime} \mathrm{O}\end{array}$ & Cambissolo & Floresta em encosta & $\mathrm{O}$ \\
\hline 2B2 & 1740 & $\begin{array}{c}28^{\circ} 07^{\prime} 02^{\prime \prime} \mathrm{S} \mathrm{e} \\
49^{\circ} 29^{\prime} 59^{\prime \prime} \mathrm{O}\end{array}$ & Neossolo Litólito & Floresta em encosta & $\mathrm{O}$ \\
\hline $3 \mathrm{C} 6$ & 1760 & $\begin{array}{l}28^{\circ} 06^{\prime} 57^{\prime \prime} \mathrm{S} \mathrm{e} \\
49^{\circ} 30^{\prime} 01^{\prime \prime} \mathrm{O}\end{array}$ & Cambissolo & Floresta em encosta & $\mathrm{O}$ \\
\hline $3 \mathrm{C7}$ & 1750 & $\begin{array}{c}28^{\circ} 06^{\prime} 55^{\prime} \text { 'S e } \\
49^{\circ} 30^{\prime} 01^{\prime \prime} \mathrm{O}\end{array}$ & Cambissolo & Floresta em encosta & $\mathrm{O}$ \\
\hline 4A8 & 1720 & $\begin{array}{c}28^{\circ} 06^{\prime} 51^{\prime \prime} \mathrm{S} \mathrm{e} \\
49^{\circ} 30^{\prime} 05^{\prime} \mathrm{O}\end{array}$ & Cambissolo & Floresta em encosta & $\mathrm{FO}$ \\
\hline
\end{tabular}

Fonte: Autores (2016)

Em que: Códigos: Estágios sucessionais - 1 = Estágio inicial ou fase de iniciação, 2 = Estágio jovem ou fase de exclusão competitiva, 3 = Estágio transição ou fase de reinício do sub-bosque, 4 = Estágio avançado/maduro ou fase de dinâmica de clareiras. Intensidades de perturbação por pastoreio - A = abandono/sem registro de pastoreio - sub-bosque denso (regeneração natural e pequenas árvores) e sem registros visuais de caminhamento e fezes bovinas (1A1) ou apenas trilhas antigas de caminhamento (4A8); $\mathrm{B}$ = pouco pastoreio - sub-bosque com pouca vegetação (regeneração natural e pequenas árvores) e poucos registros visuais de trilhas por caminhamento animal e fezes; $\mathrm{C}=$ médio pastoreio - subbosque com pouca vegetação (regeneração natural e pequenas árvores), mas registros visuais frequentes de trilhas de caminhamento e de fezes em toda a área; $\mathrm{D}$ = pastoreio intenso - sub-bosque praticamente sem vegetação, apenas árvores adultas, registro de trilhas ativas por caminhamento e fezes frequentes em toda a área., B, C e D ver texto acima. Alt.- altitude (m.n.m.), Declividade - suave ondulado (SO) 3 - 8\%; ondulado (O) 8 - 20; forte ondulado (FO) 20 $-45 \%$.

Em todos os estandes escolhidos foram instaladas parcelas de $1.000 \mathrm{~m}^{2}$ subdividas em 10 subparcelas de $10 \mathrm{~m} \times 10 \mathrm{~m}\left(\mathrm{Cl} .2=100 \mathrm{~m}^{2}\right)$, sendo no interior de cada uma destas instaladas microparcelas de $3 \times 1 \mathrm{~m}\left(\mathrm{Cl} .1=3 \mathrm{~m}^{2}\right)$. Todos os estandes apresentaram dossel contínuo (com nenhuma ou mínima abertura do dossel), longe de bordas e áreas com afloramentos de rocha ou cursos de água. Nas subparcelas de $100 \mathrm{~m}^{2}$ foram amostradas e medidas as alturas de todas as espécies arbóreas (arbustos e árvores) com o DAP $\geq 1,9 \mathrm{~cm}$ a 1,3 $\mathrm{m}$ de altura do solo. Nas microparcelas de $3 \mathrm{~m}^{2}$ foram amostradas todas as espécies arbóreas com alturas $\geq 0,2 \mathrm{~m}$ e DAP 
$<1,9 \mathrm{~cm}$. O tamanho de $1.000 \mathrm{~m}^{2}$ das parcelas foi suficiente para manter uniforme a fisionomia da vegetação, o nível de impacto e reduzir a variação espaço-temporal das variáveis físicas e biológicas (FRELICH, 2002). As espécies arbóreas (árvores e arbustos) amostradas foram enquadradas em famílias conforme APG IV (ANGIOSPERM PHYLOGENY GROUP, 2016).

\section{Análise dos dados}

As relações de similaridades florísticas entre os estandes para as duas classes de tamanho de plantas $(\mathrm{Cl} .1=\mathrm{h}>0,2 \mathrm{~m}$ e DAP $<1,9 \mathrm{~cm}$ e Cl.2 = DAP $\geq 1,9 \mathrm{~cm})$ foram avaliadas com o método de agrupamento, usando dados de presença e ausência utilizando o coeficiente de Jaccard no cálculo de similaridade e o método de ligação média entre grupos. A estrutura das comunidades arbóreas nos estandes para a Cl.1 foram representados por seis variáveis estruturais e por 14 variáveis estruturais na Cl.2. As estruturas das populações nos estandes foram comparadas pelos valores de densidade relativa (DR) na Cl.1 e pelo valor de importância (IVI\%) na Cl.2. Todas as análises foram realizadas com o pacote estatístico FITOPAC (SHEPHERD, 2006).

\section{Resultados}

\section{A composição e relações florísticas}

A lista florística das espécies amostradas nos oito estandes de florestas nebulares ( 0,8 ha) foi composta por 47 espécies (sendo duas até o nível de gênero e mais 2 táxons não identificados e encaminhados para especialistas), divididas em 28 gêneros e 23 famílias (Tabela 2).

Tabela 2 - Lista florística das espécies arbóreas (regenerantes e estabelecidos) amostradas em oito estandes de floresta nebular com pastoreio bovino no Parque Nacional de São Joaquim - SC

Table 2 - List of woody species found in the cloud forests with different successional stages and levels of cattle grazing in São Joaquim National Park, Santa Catarina state, Brazil

\begin{tabular}{|c|c|c|}
\hline Espécies & Código & Família \\
\hline Schinus polygamus (Cav.) Cabrera & Sch. pol. & Anacardiaceae \\
\hline Ilex kleinii Edwin. & Ile. kle. & Aquifoliaceae \\
\hline Ilex microdonta Reissek. & Ile. mic. & Aquifoliaceae \\
\hline Baccharis semiserrata DC. & Bac. sem. & Asteraceae \\
\hline Baccharis trimera (Less.) DC. & Bac. tri. & Asteraceae \\
\hline Baccharis uncinella DC. & Bac. unc. & Asteraceae \\
\hline Baccharis sp.1 & Bac. sp. & Asteraceae \\
\hline Campovassoura sp. & Cam. sp. & Asteraceae \\
\hline Dasyphyllum spinescens (Less.) Cabrera & Das. spi. & Asteraceae \\
\hline Symphyopappus itatiayensis (Hieron.) R.M. King \& H.Rob. & Sym. ita. & Asteraceae \\
\hline Berberis laurina Thunb. & Ber. lau. & Berberidaceae \\
\hline Citronella paniculata (Mart.) Howard & Cit. pan. & Cardiopteridaceae \\
\hline Maytenus boaria Molina & May. boa. & Celstraceae \\
\hline
\end{tabular}

Ci. Fl., Santa Maria, v. 30, n. 1, p. 1-17, jan./mar. 2020 
Tabela 2 - Conclusão ...

Table 2 - Conclusion ...

\begin{tabular}{|c|c|c|}
\hline Espécies & Código & Família \\
\hline Clethra uleana Sleumer & Cle. ule. & Clethraceae \\
\hline Weinmannia humilis Engl. & Wei. hum. & Cunoniaceae \\
\hline Dicksonia sellowiana Hook. & Dic. sel. & Dicsoniaceae \\
\hline Crinodendron brasiliense Reitz \& L.B.Sm & Cri. bra. & Elaeocarpaceae \\
\hline Escalloniapetrophila Rambo \& Sleumer & Esc. pet. & Escalloniaceae \\
\hline Mimosa scabrella Benth. & Mim. sca. & Fabaceae \\
\hline Ocoteacorymbosa (Meissn.) & Oco. cor. & Lauraceae \\
\hline Ocotea pulchellaNees et Mart. ex Nees & Oco. pul. & Lauraceae \\
\hline Leandra sp.1 & Lea. sp1. & Melastomataceae \\
\hline Leandra sp.2 & Lea. sp2. & Melastomataceae \\
\hline Myrceugenia alpigena (DC.) Landrum & Myr. alp. & Myrtaceae \\
\hline Myrceugenia euosma (O. Berg) D. Legrand & Myr. euo. & Myrtaceae \\
\hline Myrceugenia glaucescens (Cambess.) & Myr. gla. & Myrtaceae \\
\hline Myrceugenia mesomischa (Burret) D. Legrand et Kausel & Myr. mes. & Myrtaceae \\
\hline Myrceugenia miersiana (Gardner) D. Legrand \& Kausel & Myr. mie. & Myrtaceae \\
\hline Myrceugenia myrcioides (Cambess.) & Myr. myr. & Myrtaceae \\
\hline Myrceugenia ovata (Hook. \&Arn.) O. Berg. & Myr. ova. & Myrtaceae \\
\hline Myrceugenia oxysepala (Burret) D. Legrand \& Kausel. & Myr. oxy. & Myrtaceae \\
\hline Myrceugenia pilotantha (Kiaersk.) Landrum. & Myr. pil. & Myrtaceae \\
\hline Myrrhiniuma tropurpureum Schott & Myr. atr. & Myrtaceae \\
\hline Siphoneugena reitzzi D. Legrand. & Sip. rei. & Myrtaceae \\
\hline Chusquea mimosa McClure \& L.B. Sm. & Chu. mim. & Poaceae \\
\hline Myrsine coriaceae (Sw.) R.Br. & Myr. cor. & Primulaceae \\
\hline Roupala brasiliensis Klotzsch. & Rou. bra. & Proteaceae \\
\hline Prunus myrtifolia (L.) Urb. & Pru. myr. & Rosaceae \\
\hline Xylosma tweediana (Clos) Eichler & Xyl. twe. & Salicaceae \\
\hline Solanum cassioides L.B.Sm. \&Downs & Sol. cas. & Solanaceae \\
\hline Solanum paranense Dusén & Sol. par. & Solanaceae \\
\hline Solanumreitzii L.B. Sm. \&Downs & Sol. rei. & Solanaceae \\
\hline Solanum sanctaecatharinae Dunal. & Sol. cat. & Solanaceae \\
\hline Symplocos pentandra (Mattos) Occhioni & Sym. pen. & Symplocaceae \\
\hline Symplocos tetrandra (Mart.) Miq. & Sym. tet. & Symplocaceae \\
\hline Duranta vestita Cham. & Dur. ves. & Verbenaceae \\
\hline Drymis angustifólia Miers. & Dry. ang. & Winteraceae \\
\hline
\end{tabular}

Fonte: Autores (2016) 
As espécies amostradas foram comuns a outras florestas nebulares (FN) do Planalto Catarinense (FALKENBERG, 2003; MARTINS et al., 2011; HIGUCHI et al., 2013; MARCON et al., 2014) e não foi citada nenhuma espécie nova para esta região. Uma das espécies constou na Lista Oficial das Espécies da Flora Brasileira Ameaçadas de Extinção (Dicksonia sellowiana) e uma espécie é endêmica do estado de Santa Catarina (Crinodendron brasiliense) (GASPER, 2013). As famílias Myrtaceae, Symplocaceae, Melastomataceae, Aquifoliaceae que são típicas das florestas nebulares na região neotropical (WEBSTER, 1995), marcaram presença tanto na densidade como frequência de espécies, fato também evidenciado por Falkenberg (2003) na mesma região de estudo. Myrceugenia, o gênero mais rico em espécie neste estudo, também foi considerado com elevada riqueza nas FN de Santa Catarina e Rio Grande do Sul (FALKENBERG, 2003) e o segundo mais rico em espécie nas florestas nebulares no estado do Paraná (SCHEER et al., 2011). Algumas espécies amostradas como Ilex microdonta, Clethra uleana, Weinmannia humilis, Citronella paniculata, Ocotea corymbosa, Drymis angustifolia, Myrceugenia ovata, Myrceugenia pilotantha e Siphoneugena reitzii também ocorrem em FN preservadas no Paraná (KOEHLER; GALVÃO; LONGHI, 2002; SCHEER et al., 2011).

O dendrograma de similaridade para a regeneração natural (Cl.1) revelou fortes associações florísticas entre as duas florestas em estágio de transição e com intensidade média de perturbação por pastoreio (3C6 e 3C7) e entre as duas florestas em estágio jovem com pastoreio intenso (2D4 e 2D5). Houve pouca relação florística entre as duas florestas com menores impactos por pastoreio (2B3 e 4A8) e as menores relações ocorreram entre as duas florestas em estágio jovem que se localizaram fora da encosta e com abandono e forte perturbação por pastoreio (1A1 e 2D2) (Figura 1A). Para o componente estabelecido $(\mathrm{Cl} .2=\mathrm{DAP} \geq 1,9 \mathrm{~cm})$, a maior similaridade florística ocorreu entre os dois estandes em estágio de transição com média intensidade de perturbação por gado (3C6 e 3C7). Todos os demais trechos apresentaram baixa associação florística, sendo que a floresta na fase inicial de sucessão (com monodominância de Baccharis uncinella) foi a mais distinta entre todas (Figura 1B).

Figura 1 - Dendrograma de similaridade florística para a regeneração natural $(A=A l t>0,2$ m e DAP $<1,9 \mathrm{~cm})$ e componente estabelecido $(B=D A P \geq 1,9 \mathrm{~cm})$ em trechos de florestas nebulares em diferentes estágios sucessionais e níveis de pastoreio no Parque Nacional de São Joaquim - SC. Códigos, ver Tabela 1

Figure 1 - Dendrograms derived of presence-ausence data of woody species in natural regeneration $(\mathrm{A}=$ height $>0,2 \mathrm{~m}$ and $\mathrm{DBH}<1,9 \mathrm{~cm})$ and poles and adult trees $(\mathrm{B}=\mathrm{DBH} \geq 1,9 \mathrm{~cm})$ found in the cloud forests with different successional stages and levels of cattle grazing in São Joaquim National Park, Santa Catarina state, Brazil. Codes see Table 1

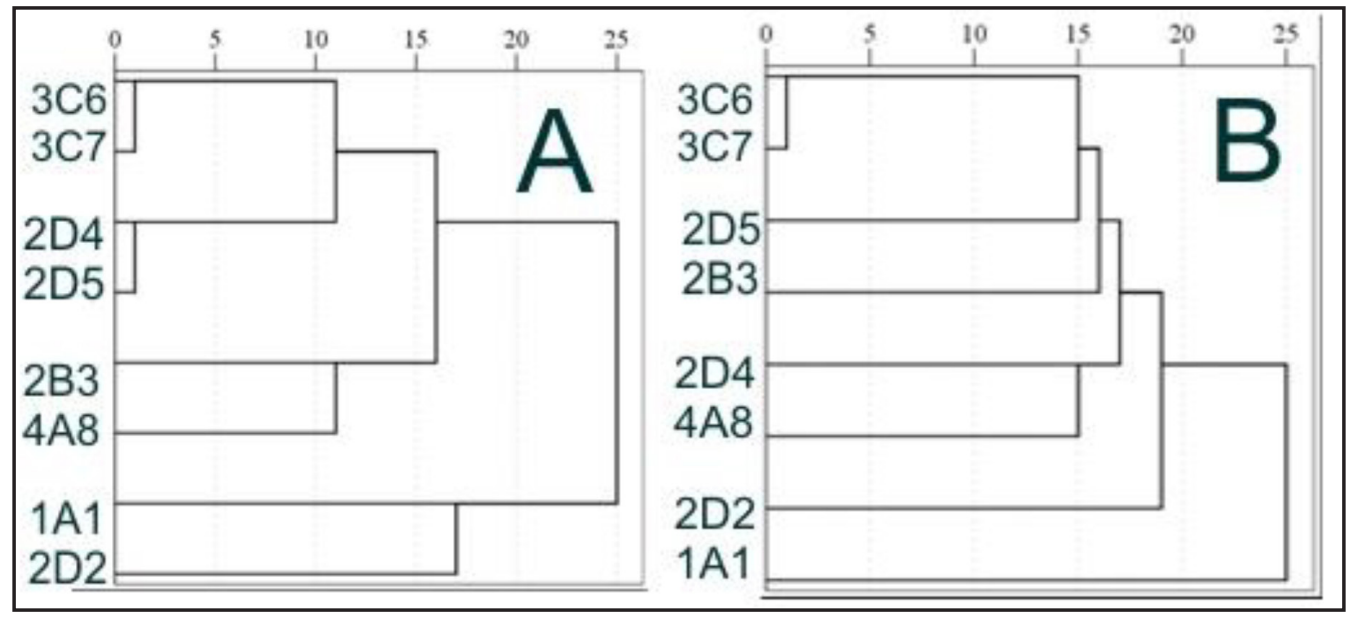

Fonte: Autores (2016) 


\section{A estrutura geral das comunidades}

$\mathrm{Na}$ Cl.1, a floresta em estágio inicial (1A1) apresentou os menores valores de riqueza, indivíduos e altura. Estes baixos valores estão relacionados com a grande cobertura de gramíneas existente, que impede o estabelecimento e desenvolvimento das sementes de espécies arbóreas (GUARIGUATA; OSTERTAG, 2001). No entanto, outras áreas jovens abandonadas na região de estudo (que não formaram vassourais monodominantes), apresentaram um maior recrutamento e desenvolvimento de espécies arbóreas (DALLABRIDA et al., 2015). Os maiores valores de riqueza, indivíduos e altura foram registrados na floresta em estágio avançado (4A8) com abandono do pastoreio. As florestas com similar estágio sucessional e nível de pastoreio também apresentaram estrutura similares entre si (2D3/2D4, exceto 2D2; 3C6 e 3C7). De maneira geral, as florestas com menor impacto por pastoreio apresentaram os maiores valores de riqueza, densidade e altura das plantas (exceto E2F2). Estes dados concordam em parte com a hipótese levantada por Falkenber (2003) de que o gado aumentaria a riqueza de espécies nas FN, visto que intensidades médias de impacto podem favorecer a riqueza e intensidades fortes de impacto podem diminuir a riqueza. O único trecho impactado (2D2) que apresentou elevados valores em riqueza e densidade de plantas (em relação aos trechos com similares estágios sucessionais e nível de impacto) foi aquele fortemente fragmentado e localizado em meio ao campo, estando sujeito à maior incidência luminosa e, consequentemente, com a regeneração natural de espécies pioneiras dependentes de luz do que aqueles no interior da encosta (GUARIGUATA; OSTERTAG, 2001).

Tabela 3 - Parâmetros estruturais para a regeneração natural $(\mathrm{h}>0,2 \mathrm{~m}$ e DAP $<1,9 \mathrm{~cm})$ nas oito parcelas amostrais em diferentes estágios sucessionais e níveis de perturbação por pastoreio bovino em florestas nebulares no Parque Nacional de São Joaquim - SC

Table 3 - Structural characteristics in natural regeneration $(\mathrm{A}=$ height $>0,2 \mathrm{~m}$ and $\mathrm{DBH}<$ $1,9 \mathrm{~cm}$ ) found in the eight cloud forests with different successional stages and levels of cattle grazing in São Joaquim National Park, Santa Catarina state, Brazil

\begin{tabular}{lcccccccc}
\hline & 1A1 & 2D5 & 2B2 & 2D3 & 2D4 & 3C6 & 3C7 & 4A8 \\
\hline N. de indivíduos & 10 & 135 & 137 & 26 & 28 & 98 & 108 & 220 \\
N. de Espécies & 5 & 12 & 18 & 7 & 7 & 14 & 11 & 23 \\
N. de Famílias & 4 & 7 & 13 & 6 & 7 & 10 & 8 & 15 \\
Mortas & 0 & 4 & 5 & 4 & 4 & 6 & 0 & 5 \\
N. de Amostras & 10 & 10 & 10 & 10 & 10 & 10 & 10 & 10 \\
Densidade (ha-1) & $3.333,3$ & 45.000 & $45.666,6$ & $8.666,6$ & $9.333,3$ & $32.666,6$ & 36.000 & $73.333,3$ \\
Área total da amostra $\left(\mathbf{m}^{2}\right)$ & 30 & 30 & 30 & 30 & 30 & 30 & 30 & 30 \\
Altura $(\text { Mín-Máx })^{(0,2-0,3)}$ & $(0,2-1,9)$ & $(0,2-2,5)$ & $(0,2-3)$ & $(0,2-0,77)$ & $(0,2-2,5)$ & $(0,2-2,7)$ & $(0,2-2,8)$ \\
\hline
\end{tabular}

Fonte: Autores (2016)

Em que: Códigos, ver Tabela 1.

Para a Cl.2 (DAP $\geq 1,9 \mathrm{~cm}$ ), a densidade de plantas variou muito entre os estandes não sendo possível estabelecer uma relação clara de impacto e/ou estágio sucessional. Mas a área basal total e a riqueza de espécies e de famílias se mantiveram maior nas florestas com menor impacto (2B3 e 4A8). A floresta mais antiga e com abandono do pastoreio também demonstrou maiores valores de altura máxima, diversidade/equabilidade e menor porcentagem de indivíduos ramificados 
(Tabela 4). A floresta antiga com abandono de pastoreio apresentou maior densidade e riqueza (Cl.1) e poderia ser usada como referência para estudos comparativos na região, pois geralmente apresenta um sub-bosque denso com ampla regeneração de espécies arbóreas (FALKENBERG, 2003). Considerando-se que são áreas de encosta, tudo leva a crer que a forte declividade e a menor acessibilidade do gado aumentariam o esforço e gasto de energia para o forrageio nesta área (TASKER; BRADSTOCK, 2006), tornando esta apenas uma área de passagem.

Os estandes com maior impacto na encosta (2D3, 2D4 e 2D5) apresentaram plantas com menor porte (diâmetro e alturas máximas) do que os estandes com menor impacto do pastoreio (Tabela 4). Maiores porcentagens de indivíduos ramificados (na base do tronco ou em pequena altura) foram registrados em estandes com maior intensidade de pastoreio e nas fases sucessionais mais jovens das florestas. Além de grande parte das espécies apresentarem naturalmente esta característica (FALKENBERG, 2003), o multiperfilhamento pode ser uma adaptação da planta para sobreviver sob o ambiente com pastoreio (ZAMORANO-ELGUETA et al., 2012) e mais comum nas fases mais jovens da floresta (KILCA, 2014). Neste estudo, as Myrceugenias (Myrceugenia euosma, Myrceugenia ovata, Myrceugenia oxysepala, Myrceugenia pilotantha) se destacaram com valores entre 30 a $80 \%$ de indivíduos ramificados, seguidos por Drymis angustifolia (dados não apresentados). As FN em estágio sucessional intermediário e com média intensidade de perturbação (MMED e MMED2) apresentaram composição florística e estrutura similares. A proximidade e a similar intensidade de pastoreio e carga animal, parecem ter conferido condições semelhantes de reprodução/regeneração das espécies (FRELICH, 2002).

No geral, foram observadas muitas diferenças florísticas e estruturais para as FN em diferentes estágios e níveis de perturbação da mesma maneira como ocorre em outras florestas temperadas (FRELICH, 2002; 2016) ou tropicais (CHAZDON, 2008). Os resultados demonstraram que o pastoreio bovino afetou pouco a composição e a estrutura das comunidades na $\mathrm{Cl} .2 \mathrm{em}$ relação à Cl.1. Outros estudos também evidenciaram maiores mudanças na densidade, riqueza e porte das espécies no componente regenerativo e no herbáceo das florestas com pastoreio bovino do que no componente adulto da vegetação (BELSY; BLUMANTHAL, 1997; TASKEY; BRADSTOCK, 2006; ZAMORANO-ELGUETA et al., 2012; KILCA, 2014). Assim, os efeitos múltiplos do pastoreio no componente regenerativo, como o forrageamento, o pisoteio e o caminhamento no sub-bosque podem ter selecionado um grupo de espécies com nichos ambientais similares para cada nível de impacto (McEVOY et al., 2006).

\section{Tabela 4 - Parâmetros estruturais para a comunidade arbórea (DAP $\geq 1,9 \mathrm{~cm})$ em trechos com diferentes estágios sucessionais e níveis de perturbação por pastoreio bovino em florestas nebulares no Parque Nacional de São Joaquim - SC}

Table 4 - Structural characteristics for woody species $(\mathrm{DBH} \geq 1,9 \mathrm{~cm})$ found in the eight cloud forests with different successional stages and levels of cattle grazing in São Joaquim National Park, Santa Catarina state, Brazil

\begin{tabular}{lcccccccc}
\hline & 1A1 & 2D5 & 2D3 & 2D4 & 2B2 & 3C6 & 3C7 & 4A8 \\
\hline No. de indivíduos & 597 & 208 & 494 & 287 & 616 & 231 & 238 & 264 \\
No. de Espécies & 1 & 15 & 23 & 13 & 27 & 19 & 18 & 32 \\
No. de Famílias & 1 & 7 & 13 & 9 & 16 & 10 & 12 & 18 \\
No. de Amostras & 10 & 10 & 10 & 10 & 10 & 10 & 10 & 10 \\
Densidade $\left(\right.$ ha $\left.^{-1}\right)$ & 5970 & 2080 & 4940 & 2870 & 6160 & 2310 & 2380 & 2640
\end{tabular}


Tabela 4 - Conclusão ...

Table 4 - Conclusion ...

\begin{tabular}{lcccccccc}
\hline & 1A1 & 2D5 & 2D3 & 2D4 & 2B2 & 3C6 & 3C7 & 4A8 \\
\hline Área Basal total & 8,19 & 1,65 & 6,63 & 6,98 & 10,35 & 7,63 & 7,05 & 5,14 \\
Área total da amostra & 0,1 & 0,1 & 0,1 & 0,1 & 0,1 & 0,1 & 0,1 & 0,1 \\
Diâmetro (Mín-Máx) & $1,9-6,17$ & $1,9-22,02$ & $1,9-32,46$ & $1,9-59,52$ & $1,9-45,83$ & $1,9-57,93$ & $1,9-61,75$ & $1,9-50,61$ \\
Altura (Mín-Máx) & $1,7-3,5$ & $1,5-6$ & $0,5-5,3$ & $1,4-5,5$ & $1,4-7,5$ & $1,4-7$ & $1,4-6,7$ & $1,4-8,2$ \\
No. total de Ramos & 1875 & 458 & 1585 & 875 & 2019 & 422 & 393 & 449 \\
No. de ind. ramificados & 409 & 104 & 308 & 164 & 367 & 89 & 84 & 74 \\
Porcentagem ramificado & 68,5 & 50 & 62,3 & 57,1 & 59,5 & 38,5 & 35,2 & 28 \\
No. de ramos & 3,1 & 2,2 & 3,2 & 3 & 3,2 & 1,8 & 1,6 & 1,7 \\
Índice Shannon-Wiener & 0,391 & 1,697 & 1,423 & 1,629 & 1,693 & 2,134 & 1,996 & 2,802 \\
Equabilidade & 0,564 & 0,627 & 0,454 & 0,635 & 0,514 & 0,725 & 0,691 & 0,808 \\
Índice Simpson & 2 & 0,298 & 0,378 & 0,258 & 0,341 & 0,162 & 0,192 & 0,092 \\
\hline
\end{tabular}

Fonte: Autores (2016)

Em que: Códigos, ver Tabela 1.

Contudo, o impacto no componente regenerativo foi significativo, pois tende a retardar a dinâmica natural do ecossistema (BELSY; BLUMENTHAL, 1997). O aumento do pastoreio bovino também influenciou na maior proporção de árvores mortas em pé, fato também evidenciado por Falkenberg (2003), e nos baixos valores de área basal devido à menor densidade de plantas nas áreas fortemente impactadas.

Os dados de riqueza de espécies na área de estudo permitem inferir uma menor riqueza do que as florestas nebulares no estado do Paraná e sudeste do Brasil (SCHEER et al., 2011; POMPEU et al., 2014). As maiores alturas das árvores nesta floresta foram similares às FN da região sul e sudeste, que raramente ultrapassam $8 \mathrm{~m}$ de altura. Os valores máximos de diâmetros do caule foram maiores que muitos estudos (KOEHLER; GALVÃO; LONGHI, 2002; SCHEER et al., 2011), embora possam existir FN em estágio avançado com maiores valores de diâmetros (ver MARCON et al., 2014). No entanto, a elevada área basal total amostrada em alguns estandes, que foram maiores que outras FN (KOEHLER; GALVÃO; LONGHI, 2002; SCHEER et al., 2011; HIGUCHI et al., 2013; MARCON et al., 2014), se deve ao critério amostral adotado que incluiu um grande número de arbóreas jovens.

\section{As espécies mais importantes}

Várias espécies se destacaram por se regenerar (Cl.1) sob a maioria das intensidades de impacto de pastoreio e estágio sucessional, como Symphyopappus itatiayensis, Prunus myrtifolia, Myrsine coriacea, Symplocos tetrandra e Myrceugenia euosma e Myrceugenia ovata por ocorrerem em mais de cinco ( $\geq 5$ estandes) florestas, (Tabela 5). Por outro lado, Chusquea mimosa, Citronela paniculata, Myrceugenia myrcioides e Myrceugenia oxysepala apresentaram maiores densidades em estandes com menores níveis de pastoreio (Tabela 5) e Myrceugenia ovata e Symplocos pentandra nas florestas mais jovens. No geral, houve fortes mudanças nas espécies mais representativas do componente regenerativo em cada floresta (Tabela 5).

Falkenberg (2003) levantou a hipótese de Symplocos spp. ocorrer em maiores densidades nas áreas com impacto por ser uma espécie impalatável ao gado, mas os dados deste estudo não 
corroboram a observação do autor. O mesmo autor também observou grandes densidades de taquaras (Chusquea mimosa) nas florestas preservadas, em que o gado atuaria como controlador desta espécie em FN com pastoreio. Visitas em outras FN abandonadas pelo pastoreio por décadas no PARNA-SJ apresentaram grande densidade de taquaras, tornando-as praticamente florestas intransponíveis (sem causar impacto relevante) para realização demais inventários para este estudo.

Tabela 5 - Valores de densidade relativa (DR\%) para as espécies arbóreas (Alt $>0,2 \mathrm{~m}$ e DAP $<1,9 \mathrm{~cm}$ ) em florestas nebulares com diferentes estágios sucessionais e níveis de impacto no Parque Nacional de São Joaquim - SC

Table 5 - Relative density (DR\%) for woody species in natural regeneration understory (height $>$ $0,2 \mathrm{~m}$ and $\mathrm{DBH}<1,9 \mathrm{~cm}$ ) in cloud forests with different successional stages and levels of cattle grazing in São Joaquim National Park, Santa Catarina state, Brazil

\begin{tabular}{|c|c|c|c|c|c|c|c|c|}
\hline Espécies & 1A1 & 2D5 & 2D3 & 2D4 & 2B2 & $3 \mathrm{C} 6$ & $3 \mathrm{C} 7$ & 4A8 \\
\hline Arv.mor. & & 3 & 17,4 & 16 & 3,67 & 6,12 & & 3,1 \\
\hline Bac. $s p$ & & & & & 4,44 & & & \\
\hline Bac. sem. & 10 & 12,7 & & & 1,47 & & & \\
\hline Bac.tri. & 20 & 27,8 & & & & & & \\
\hline Bac.unc. & & 7,51 & & & & & & \\
\hline Berlau. & & 1,54 & & & 0,73 & 1,02 & & \\
\hline Chu.mim. & & 0,75 & & & & & & 22,2 \\
\hline Cit.pan. & & & & & 2,24 & 9,18 & 3,74 & 4,32 \\
\hline Dic. sel. & & & & & & & & 3,09 \\
\hline Dry.ang. & & 6,76 & & 4 & 13,23 & 5,1 & & \\
\hline Durves. & & & & & & & & 1,23 \\
\hline Ile. mic. & 10 & 0,75 & & & 1,47 & & & \\
\hline Lea. sp.1 & 40 & & & & 11,02 & & 8,41 & 8,02 \\
\hline Lea. sp. 2 & & 0,75 & & & & & & 1,23 \\
\hline Liana & & 0,75 & 8,69 & & 4,41 & 1,02 & & 0,62 \\
\hline May.boa. & & & & & & & & 2,47 \\
\hline Mim.sca. & & & & & 1,47 & & & \\
\hline Myr.euo. & & 18,8 & & 4 & & 20,41 & 2,8 & 3,70 \\
\hline Myr.glau. & & & & & 4,41 & & 28,04 & \\
\hline Myr.mie. & & & & & & & & 1,23 \\
\hline Myr.myr. & & & & & & & & 11,11 \\
\hline Myr.ova. & & 14,28 & 13,4 & & 11,76 & 8,16 & 20,56 & 2,47 \\
\hline Myr.oxy. & & & & & 0,73 & & 0,93 & 4,94 \\
\hline Myr.pil. & & 4,61 & & & & 7,14 & & 0,62 \\
\hline Myr. cor. & 20 & & 17,4 & 4 & 2,94 & 8,16 & 8,41 & 2,47 \\
\hline
\end{tabular}


Tabela 5 - Conclusão ...

Table 5 - Conclusion ...

\begin{tabular}{|c|c|c|c|c|c|c|c|c|}
\hline Espécies & 1A1 & 2D5 & 2D3 & 2D4 & $2 \mathrm{~B} 2$ & $3 \mathrm{C} 6$ & $3 \mathrm{C7}$ & $4 \mathrm{~A} 8$ \\
\hline$N I$ & & & & & & & & 0,62 \\
\hline Oco. cor. & & & & & & & & 0,62 \\
\hline Oco.pul. & & & 4,33 & & 0,73 & & & 0,62 \\
\hline Pru.myr. & & & 8,69 & 12 & 1,47 & 3,06 & 3,74 & 4,94 \\
\hline Rou.bras. & & & & & & 1,02 & & \\
\hline Sch. pol. & & & 4,33 & & & & & \\
\hline Sol.cas. & & & & & & & & 2,47 \\
\hline Sol. par. & & & & & & 4,08 & 11,2 & \\
\hline Sol. rei. & & & & 8 & & 8,16 & & \\
\hline Sym. ita. & & & 4,33 & 44 & 27,2 & 3,06 & 5,6 & 5,56 \\
\hline Sym. pen. & & & 21,7 & & & & & \\
\hline Sym.tet. & & & & 8 & 5,88 & 14,29 & 6,54 & 11,73 \\
\hline Wei.hum. & & & & & 0,73 & & & \\
\hline Xyl.twe. & & & & & & & & 0,62 \\
\hline
\end{tabular}

Fonte: Autores (2016)

Em que: Códigos das áreas ver Tabela 1. Nomes das espécies ver Tabela 2.

Para o componente arbóreo estabelecido (DAP $>1,9 \mathrm{~cm}$ ), com exceção da fase inicial ou de colonização (monodominância de Baccharis uncinella), existiu uma tendência de Drymis angustifolia ser a espécie dominante mais importante ao longo da sucessão florestal. Myrceugenia ovata também foi relevante por predominar nas fases iniciais (2D3, 2D5 e 2B2) e Myrceugenia euosma na fase média de sucessão (2D4, 3C6 e 3C7). A permanência de uma ou algumas espécies ao longo da sucessão das FN demonstra que esta tipologia segue padrões sucessionais distintos da maioria das florestas tropicais e subtropicais, onde ocorrem grandes mudanças florísticas ao longo do tempo (GUARIGUATA; OSTERTAG, 2001; KILCA; LONGHI, 2011). Essas três espécies são as mais comuns na região (FALKENBERG, 2003; HIGUCHI et al., 2013; MARCON et al., 2014), sendo apenas Drymis angustifolia bem representativa no estado do Paraná (SCHEER et al., 2011).

Além destas espécies, destacaram-se também, Berberis laurina, Myrceugenia oxysepala (seis estandes) e Chusquea mimosa, Symphyopappus itatiayensis (cinco estandes) com valores de importância elevados (Tabela 6). No entanto, Crinodendron brasiliense, Dicksonia sellowiana, Myrrhinium atropurpureum e Weinmannia humilis demonstraram maiores valores de importância (VIs) apenas nas florestas com baixo impacto de gado (Tabela 6). 
Tabela 6 - Valores de importância (IVI\%) para as espécies arbóreas (DAP $\geq 1,9 \mathrm{~cm}$ ) em oito florestas nebulares diferentes estágios sucessionais e níveis de impacto no Parque Nacional de São Joaquim - SC

Table 6 - Importance value index (IVI\%) for woody species $(\mathrm{DBH} \geq 1,9 \mathrm{~cm}$ ) in eight cloud forests with different successional stages and levels of cattle grazing in São Joaquim National Park, Santa Catarina state, Brazil

\begin{tabular}{|c|c|c|c|c|c|c|c|c|}
\hline Espécies & 1A1 & 2D5 & 2D5 & 2D4 & 2B2 & $3 \mathrm{C} 6$ & $3 \mathrm{C} 7$ & 4A8 \\
\hline Arv. mor. & 21,43 & 20,50 & 8,30 & 12,58 & 5,50 & 9,42 & 10,33 & 8,54 \\
\hline Bac. sp. & & 11,09 & & & & & 0,63 & \\
\hline Bac. sem. & & & & 0,96 & 4,61 & 3,32 & 0,63 & \\
\hline Bac. unc. & 78,57 & 1,41 & & & & & & \\
\hline Bac. unc.cf & & 0,83 & & & & & & \\
\hline Ber. lau. & & 5,26 & 1,23 & 1,70 & 1,80 & 2,30 & 0,63 & \\
\hline Camp. sp. & & 1,20 & & & 0,82 & & & \\
\hline Chu. mim. & & & 1,71 & 0,79 & 0,81 & & 2,58 & 3,68 \\
\hline Lia. mor. & & & 1,09 & & 1,20 & 0,57 & & \\
\hline Cit.pan. & & & & & & & & 2,04 \\
\hline Cle. ule. & & 1,42 & & & 2,13 & & & 0,90 \\
\hline Cri. bras. & & & & & 0,40 & & & 3,15 \\
\hline Das. spi. & & & & & & & & 0,43 \\
\hline Dic. sel. & & & & & 0,97 & & & 1,46 \\
\hline Dry. ang. & & 9,32 & 29,01 & 21,17 & 16,27 & 28,19 & 32,79 & 27,33 \\
\hline Dur. ves. & & & & & & 1,87 & & \\
\hline Ile. kle. & & & & & & & 2,80 & 1,50 \\
\hline Ile. kle cf. & & & & & & & 1,88 & 2,42 \\
\hline Ile. mic. & & & & & & & 1,29 & \\
\hline Liana & & & 1,09 & 1,57 & 0,44 & 2,31 & 2,50 & 2,15 \\
\hline May. boa. & & & 0,67 & & & 2,60 & & 5,20 \\
\hline Mim. sca. & & & & & 1,51 & & 0,62 & 2,71 \\
\hline Myr. alp. & & 3,93 & & & 1,21 & & & \\
\hline Myr. euo & & 13,9 & 4,28 & 34,13 & 5,85 & 23,59 & 21,38 & 1,43 \\
\hline Myr. gla & & & 0,64 & & 0,76 & & & 4,03 \\
\hline Myr. mes & & & 1,10 & & & & & 0,43 \\
\hline Myr. mie & & 2,73 & 1,21 & 0,80 & & & & 1,32 \\
\hline Myr. myr & & & & & 0,55 & & & \\
\hline Myr. ova & & 25,3 & 38,38 & 16,18 & 45,12 & 13,01 & 10,72 & 0,86 \\
\hline Myr. oxy & & & 3,84 & 6,61 & 1,71 & 5,03 & 2,31 & 7,30 \\
\hline
\end{tabular}


Tabela 6 - Conclusão ...

Table 6 - Conclusion ...

\begin{tabular}{|c|c|c|c|c|c|c|c|c|}
\hline Espécies & 1A1 & 2D5 & 2D5 & 2D4 & 2B2 & $3 \mathrm{C} 6$ & $3 \mathrm{C} 7$ & 4A8 \\
\hline Myr. pil & & & & & 0,38 & & & 0,44 \\
\hline Myr.atr & & & & & & 1,18 & 0,62 & 1,59 \\
\hline Myr. cor & & & & 1,80 & 2,16 & 0,59 & & \\
\hline NI & & & 0,71 & 0,79 & 0,79 & & & \\
\hline Oco.pul & & & 0,56 & & & & 2,99 & 2,42 \\
\hline Pru. myr & & & 1,12 & & & & 3,15 & 0,44 \\
\hline Sch. pol & & & & & 2,16 & & & \\
\hline Sip. Rei & & & 0,55 & & 0,38 & & & \\
\hline Sol.cas & & & & & & 1,32 & 2,15 & 5,85 \\
\hline Sol.par & & 0,78 & & & & & & \\
\hline Sol. Rei & & & 0,56 & & & 0,77 & & 0,45 \\
\hline Sol. san & & & 1,11 & & & 1,19 & & 2,26 \\
\hline Sym. ita & & 1,54 & 1,64 & 0,90 & 1,70 & 1,45 & & \\
\hline Sym. pen & & & 0,65 & & & & & \\
\hline Sym.tet & & 0,76 & & & 0,38 & 0,57 & & 4,07 \\
\hline Wei. hum & & & & & & 0,73 & & 5,60 \\
\hline Xyl. twe & & & 0,55 & & 0,38 & & & \\
\hline
\end{tabular}

Fonte: Autores (2016)

Em que: Códigos das áreas ver Tabela 1. Nomes das espécies ver Tabela 2.

O fato de existir um bom número de espécies arbóreas nas florestas nebulares adaptadas ao pastoreio bovino (FALKENBERG, 2003) pode representar a mesma característica evolutiva das espécies arbóreas em outras fitofisionomias tropicais de baixa altitude (GUARIGUATA; OSTERTAG, 2001). Neste estudo, as florestas com níveis baixos ou médios de perturbação apresentaram composição e estrutura similares entre si, o que indicaria um bom e rápido potencial de recuperação desta vegetação. Assim, o emprego de técnicas de restauração passiva, como a exclusão e/ou pousio, em áreas com médias e baixas intensidades de pastorio bovino poderiam contribuir para a manutenção da diversidade vegetal nas florestas nebulares.

No entanto, altas intensidades de pastoreio podem diminuir a abundância das espécies (MCEVOY et al., 2006), como pode ter sido os casos das espécies Chusquea mimosa, Citronella paniculata, Myrceugenia glaucescens e Myrceugenia oxysepala (na regeneração natural - DAP $<1,9 \mathrm{~cm}$ ) e Crinodendron brasiliense, D. sellowiana, Myrrhinium atropurpureum, Weinmania humilis e as espécies de Ilex spp. (componente adulto). Falkenberg (2003) chamou a atenção da ocorrência de florestas nebulares com elevado grau de perturbação por pastoreio bovino na região dos Aparados (mesma região deste estudo) e à falta de registros de remanescentes florestais preservados em estado maduro para servirem de referência em estudos comparativos de impacto ou sucessão. Os resultados apresentados neste estudo também ficam limitados às comparações entre estandes em estágios sucessionais diferentes e com níveis de pastoreio baixo (ou abandonado), médio e elevado, determinados de forma subjetiva, pois não foram encontradas áreas de referência (mesmo estágio 
sucessional) sem nenhum tipo de impacto. Outros esclarecimentos sobre o impacto do pastoreio em florestas nebulares serão obtidos em inventários padronizados em cronossequências com maior número de réplicas (idades) e/ou estudos experimentais com a inserção ou exclusão do gado em fragmentos (com maior controle possível das variáveis ambientais). Assim, os resultados obtidos poderão determinar metodologias que nortearão o uso mais sustentável das florestas nebulares na região do Planalto Catarinense.

\section{Conclusão}

As espécies arbóreas amostradas na área de estudo foram comuns às outras florestas nebulares do Planalto Catarinense e diversas espécies são adaptadas à perturbação por pastoreio. A floresta com abandono do pastoreio e em estágio sucessional mais avançado diferiu das outras florestas em estágio mais jovens quanto à composição florística, maior riqueza de espécies, altura máxima, diversidade e menor porcentagem de plantas ramificadas. As florestas secundárias variaram muito de estrutura devido aos distintos níveis de pastoreio bovino. De uma maneira geral, os estágios secundários das FN apresentaram melhor capacidade de regeneração natural devido à diminuição da carga animal, da frequência de pastoreio ou com o abandono da atividade (pousio). O nível de impacto e o estágio sucessional determinaram a composição e estrutura das florestas nebulares em pequena escala espacial e, por isso, são variáveis fundamentais para delinear os estudos que busquem a sustentabilidade nas áreas de Reserva Legal das propriedades agrícolas.

\section{Agradecimentos}

O estudo foi financiado pela bolsa PNPD/CAPES/UDESC do primeiro autor. Agradecemos aos funcionários do PARNA-SJ e ICMBio por permitirem as coletas de dados e uso das dependências da UC. Ao CNPq, pela bolsa de produtividade em pesquisa do segundo e terceiro autores.

\section{Referências}

ANGIOSPERM PHYLOGENY GROUP. An update of the Angiosperm Phylogeny Group classification for the orders and families of flowering plants: APG IV. Botanical Journal of the Linnean Society, Londres, v. 181, n. 1, p. 1-20, 2016.

BELSKY, A. J.; BLUMENTHAL, D. M. Effects of livestock grazing on stand dynamics and soils in upland forests of the interior west. Conservation Biology, Washington, v. 11, n. 2, p. 315-327, abr. 1997.

BRASIL. Lei no 12.651, de 25 de maio de 2012. Disponível em: http://www.planalto.gov.br/ ccivil_03/_Ato2011-2014/2012/Lei/L12651.htm. Acesso em: 12 dez. 2015.

BRASIL. Lei no 13.273, de 15 de abril de 2016. Disponível em: http://www.planalto.gov.br/ ccivil_03/_Ato2015-2018/2016/Lei/L13273.htm. Acesso em: 30 ago. 2016.

BRASIL. Resolução CONAMA no 4, de 4 de maio de 1994. Disponível em: http://www.mma. gov.br/ port/conama/legislacao/CONAMA_RES_CONS_1994_004.pdf. Acesso em: 12 dez. 2015. 
BUBB, P. et al. Cloud Forest Agenda. Cambridge: UNEP; WCMC, 2004. 36 p.

CHAZDON, R. L. Chance and determinism in tropical forest succession. In: CARSON, W. P.; SCHNITZER, S. A. (ed.). Tropical forest community ecology. Oxford: Wiley-Blackwell, 2008. p. 384-408.

DALLABRIDA, J. P. et al. Spatial distribution of regenerating tree species in an area of forestgrassland transition in Southern Brazil. In: WORLD FORESTRY CONGRESS, 13., Durban, South of Africa, 2015. Anais [...]. Durban: [s. n.], 2015. v. 2. p. 94.

FALKENBERG, D. B. Matinhas nebulares e vegetação rupícola dos Aparados da Serra Geral (SC/RS), sul do Brasil. 2003. Tese (Doutorado em Biologia Vegetal) - Universidade Estadual de Campinas, Campinas, 2003.

FALKENGERG, D. B.; VOLTOLinI, J. C. The montane cloud forest in Southern Brazil. In: HAMILTON, L. S. et al. (ed.). Tropical montane cloud forest. New York: Springer-Verlag, 1995. p. $138-149$.

FAXINA, T.C. Dilemas da regularização fundiária no parquet nacional de São Joaquim: a valoração de áreas naturais. 2014. Dissertação (Mestrado em Engenharia Florestal) - Universidade do Estado de Santa Catarina, 2014.

FOSTER, P. The potential negative impacts of global climate change ontropical montane cloud forests. Earth-Science Reviews, New York, v. 55, p. 73-106, out. 2001.

FRELICH, L. E. Forest dynamics and disturbance regimes: studies from temperate evergreendeciduous forest. Cambridge: Cambridge University Press, 2002. 278 p.

FRELICH, L. Forest Dynamics. F1000Research, [s. l.], n. 5, p. 183. 2016. doi: https://doi. org/10.12688/f1000research.7412.1.

GASPER, A. L. et al. Inventário Florístico Florestal de Santa Catarina: espécies da Floresta Ombrófila Mista. Rodriguésia, Rio de Janeiro, v. 64, n. 3, p. 427-443, jul./set. 2013.

GUARIGUATA, M.R.; OSTERTAG, R. Neotropical secondary forest succession: changes in structural and functional characteristics. Forest ecology and management, Amsterdam, v. 148, n. 1-3, p. 185-206, 2001.

HIGUCHI, P. et al. Florística e estrutura do componente arbóreo e análise ambiental de um fragmento de Floresta Ombrófila Mista Alto-Montana no município de Painel, SC. Ciência Florestal, Santa Maria, v. 23, n. 1, p. 153-164, jan./mar. 2013.

IBGE. Manual Técnico da Vegetação Brasileira. Rio de Janeiro, 2012. 271 p.

KILCA, R. V. A sucessão secundária na floresta estacional subtropical do Rio Grande do Sul, Brasil. 2014. Tese (Doutorado em Engenharia Florestal) - Universidade Federal de Santa Maria, Santa Maria, 2014.

KILCA, R. V.; LONGHI, S. J. A composição florística e a estrutura das florestas estacionais subtropicais secundárias nas escarpas do Planalto Meridional do Rio Grande do Sul. In: SCHUMACHER, M. V. et al. (org.). A Floresta Estacional Subtropical: carcterização e ecologia no rebordo do Planalto Meridional. Santa Maria: Palloti, 2011. p. 55-83.

KOEHLER, A.; GALVÃO, F.; LONGHI, S. J. Floresta Ombrófila Densa Altomontana: aspectos florísticos e estruturais de diferentes trechos da serra do mar. Ciência Florestal, Santa Maria, v. 12, n. 2, p. 27-39, 2002.

MARCON, A. K. et al. Variação florístico-estrutural em resposta à heterogeneidade ambiental em uma Floresta Nebular em Urubici, Planalto Catarinense. Scientia Forestalis, Piracicaba, v. 42, n. 103, p. 439-450, set. 2014. 
MARTIN, P. H. et al. Vegetation Zonation in a Neotropical Montane Forest: Environment, Disturbance and Ecotones. Biotropica, Gainesville, v. 43, n. 5, p. 533-543, set. 2011.

MARTINS, D. et al. Florística de Floresta Ombrófila Mista Altomontana e de Campos em Urupema, Santa Catarina, Brasil. Revista Brasileira de Biociências, Porto Alegre, v. 9, n. 2, p. 156-166, abr.jun. 2011.

MCEVOY, P. M. et al. The effects of livestock grazing on ground flora in broadleaf woodlands in Northern Ireland. Forest Ecology and Management, Amsterdam, v. 225, p. 39-50, abr. 2006.

PONCE-REYES, R. et al. Extinction risk in cloud forest fragments under climate change and habitat loss. Diversity and Distributions, Arizona, v. 19, n. 5/6, p. 518-529, maio/jun. 2013.

RODERJAN, C. V. et al. As unidades fitogeográficas do estado do Paraná. Ciência e Ambiente, Santa Maria, v. 24, p. 75-92, jan./jul. 2002.

SHEPHERD, G. J. Fitopac 2.1: manual do usuário. Campinas: Universidade Estadual de Campinas, 2006.

SCHEER et al. Estrutura arbórea da Floresta Ombrófila Densa Altomontana de serras do Sul do Brasil. Acta Botanica Brasilica, Feira de Santana, v. 25, n. 4, p. 735-750, out./dez. 2011.

POMPEU, P. V. et al. Floristic composition and structure of an upper montane cloud forest in the Serra da Mantiqueira Mountain Range of Brazil. Acta Botanica Brasilica, Feira de Santana, v. 28 , n. 3, p. 456-464, jul./set. 2014.

SOUZA, B. Aspectos fitogeográficos do Parque Nacional de São Joaquim. 2004. Monografia (Bacharel em Geografia) - Universidade Federal de Santa Catarina, Florianópolis, 2004.

TASKER, E. M.; BRADSTOCK, R. A. Influence of cattle grazing practices on forest understorey structure in north-eastern New South Wales. Austral Ecology, Melbourne, v. 31, n. 5, p. 490-502, set. 2006.

WEBSTER, G. L. The panorama of Neotropical cloud forests. In: CHURCHILL, S. P. et al. (ed.) Biodiversity and conservation of Neotropical Montane Forests. New York: The New York Botanical Garden, 1995. p. 53-77.

ZAMORANO-ELGUETA, C. et al. Impacts of cattle on the South American temperate forests: challenges for the conservation of the endangered monkey puzzle tree (Araucaria araucana) in Chile. Biology and Conservation, Boston, v. 152, p. 110-118, aug. 2012. 